\title{
Combustion peculiarities of coal-methane-air mixtures in a recuperative burner
}

\author{
Leonid Minkov ${ }^{1,}$, and Kseniya Moiseeva ${ }^{1}$ \\ ${ }^{1}$ Tomsk State University, 634050, 36 Lenin av., Tomsk, Russia
}

\begin{abstract}
Numerical modeling of the combustion of a lean methane-air mixture containing fine coal particles entering the "Swiss-roll" type recuperative burner is considered. The mathematical model is constructed under the following assumptions: the flow field is two-dimensional; the gas mixture is an ideal incompressible gas consisting of oxygen, methane, coal volatile substances, carbon monoxide, carbon dioxide, water vapor, hydrogen and nitrogen. In the gas phase four oxidation reactions, in which methane, volatile matter of coal, carbon monoxide, hydrogen participate and the reaction of carbon dioxide decomposition take place. On the surface of the coal particle, there are three oxidation reactions involving oxygen, carbon dioxide and water vapor, resulting in the formation of carbon monoxide. It is assumed that coal contains $8 \%$ of ash, $12.9 \%$ of volatile substances and $79.1 \%$ of carbon. It is shown that for a two percent methane-air mixture the reaction zone shifts toward the center of the burner as the feed rate of the mixture increases. An increase in the content of coal particles leads to a shift of the reaction zone into the inlet part of the burner, and the heat release in the burner increases.
\end{abstract}

\section{Introduction}

As noted in the report [1], a large number of actually neglected potentially useful fuels will become sources of combustion energy in the future. Such fuels include gases originating from coal seams in opencast mines or exhaust gases of various industrial plants, lean methaneair mixture formed in the fermentation of domestic waste and sewage drained. Since the concentration of fuel and oxidant in such mixtures is below the limits of flammability under normal conditions, and also because the mixtures have a low calorific value, one of the methods of extracting the energy of combustion is the use of burners in which the burning rate is increased by recycling the heat between the reagents and reaction products by means of a heat exchanger [2].

Swiss-roll type helical burners, as heat sources, have several advantages over other types of burners, since they provide a number of necessary functions [3], such as: 1) transfer of thermal energy from hot combustion products to cold reagents for preliminary heating; 2) the relatively large ratio of the inner surface area of the walls to the volume of the burner, which is necessary for efficient heat transfer, as well as for the effective action of the catalyst, if applicable; 3 ) a large area of the upper and lower surface for transferring heat to external devices.

The use of micro-burners and microreactors, whose action is based on the Swiss-roll approach, as sources of high specific energy for microelectromechanical systems are discussed in [4].

A lot of work has been devoted to the investigation of combustion processes of a mixture of gaseous hydrocarbons and air in burners working on the principle of heat recovery. Thus, in Ref. [5], the effect of the thermal conductivity of the burner walls, the feed rate and composition of the methane-air mixture on the stability of its combustion in a recuperative burner of the U shape was studied on the basis of a numerical solution of the Navier-Stokes equations and the transport equations for a multicomponent mixture in the twodimensional approximation. The model took into account 9 gaseous and 11 surface components that participated in 24 reactions, including catalytic ones. Platinum was the catalyst. It is shown that the thermal conductivity of the walls plays a decisive role in the creation of this type of burner. Effect of the burner size on catalytic combustion of methane-air mixtures was studied in [6].

Analysis of the effect of methane concentration in the methane-air mixture, feed rate of the mixture and the channel size on the stable operation of the $U$ shape recovery burner in one-dimensional approximation was performed in $[7,8]$. Studies $[9,10]$ performed on the basis of two-dimensional modeling show that even in an idealized combustion chamber the behavior of combustion is rather complicated and cannot be properly described by phenomenological or one-dimensional models.

The influence of the wall material on the parameters of the "Swiss-roll" burner operating on a mixture of air and associated petroleum gas was tested experimentally in Ref. [11]. It has been shown stainless steel is a preferred material than aluminum, copper or brass, from the viewpoint of achieving the maximum temperature in the combustion chamber and it provides higher heat

Corresponding author: lminkov@ftf.tsu.ru 
content. Similar results were obtained by the authors of Ref. [12] who, based on experiments and numerical simulation, showed that the burner walls made of stainless steel provide greater heat recirculation than the walls made of quartz or silicon carbide.

A numerical study carried out on the basis of a simplified one-dimensional model of coal combustion [13-14] shows that the presence of small reacting coal particles in the methane-air mixture, which is a product of mine production, leads to an expansion of the area of stable combustion in recuperative burners, while inert particles, on the contrary, narrow the area. Reducing the size of the reacting particles also extends the range of stable combustion.

The aim of the work is to study the stable combustion modes of a methane-air mixture containing reactive coal particles in a four-turn "Swiss-roll" burner, depending on the parameters of the coal-methane-air mixture supplied, based on numerical simulation in the two-dimensional approximation.

\section{Statement of the problem}

The coal-methane-air mixture enters the "Swiss-roll" burner with the speed $U_{i n}$ and the temperature $T_{i n}$, Figure 1. It is assumed that the outer walls of the burner are thermally insulated. The cold mixture, due to the heat recovery effect from hot combustion products through the inner walls of the burner, is heated to a temperature at which the components react chemically. The heat released during this process is used to replenish the heat lost by the combustion products, which has gone to the heating of the cold mixture entering the burner.

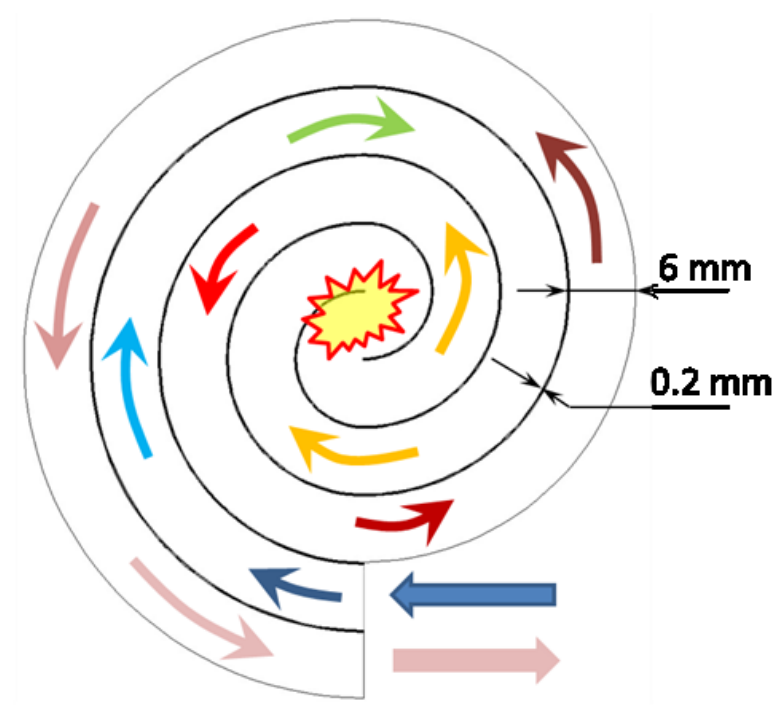

Fig. 1. Swiss-roll burner.

The combustion model of the coal-methane-air mixture is constructed under the following assumptions. Gas flow is a multicomponent viscous ideal incompressible one consisting of oxygen $\mathrm{O}_{2}$, methane $\mathrm{CH}_{4}$, coal volatile substances $\mathrm{CH}_{8.34} \mathrm{O}_{0.212}$, carbon monoxide $\mathrm{CO}$, carbon dioxide $\mathrm{CO}_{2}$, water vapor $\mathrm{H}_{2} \mathrm{O}$ hydrogen $\mathrm{H}_{2}$ and nitrogen $\mathrm{N}_{2}$. In the gas phase there are four oxidation reactions, involving methane, volatile matter of coal, carbon monoxide, hydrogen participate, and a decomposition reaction of carbon dioxide:

1) $\mathrm{CH}_{4}+1.5 \mathrm{O}_{2}=\mathrm{CO}+2 \mathrm{H}_{2} \mathrm{O}$;

2) $\mathrm{CO}+0.5 \mathrm{O}_{2}=\mathrm{CO}_{2}$;

3) $\mathrm{CO}_{2}=\mathrm{CO}+0.5 \mathrm{O}_{2}$;

4) $\mathrm{CH}_{8.34} \mathrm{O}_{0.212}+2.979 \mathrm{O}_{2}=\mathrm{CO}_{2}+4.17 \mathrm{H}_{2} \mathrm{O}$;

5) $\mathrm{H}_{2}+0.5 \mathrm{O}_{2}=\mathrm{H}_{2} \mathrm{O}$.

On the surface of the coal particle, there are three oxidation reactions involving oxygen, carbon dioxide and water vapor, resulting in the formation of carbon monoxide:

6) $\mathrm{C}<s>+0.5 \mathrm{O}_{2}=\mathrm{CO}$;

7) $\mathrm{C}<s>+\mathrm{CO}_{2}=2 \mathrm{CO}$;

8) $\mathrm{C}<\mathrm{s}>+\mathrm{H}_{2} \mathrm{O}=\mathrm{H}_{2}+\mathrm{CO}$.

It is assumed that coal contains $8 \%$ of ash, $12.9 \%$ of volatile substances and $79.1 \%$ of carbon.

The system of equations describing the parameters of the gas mixture includes

- the continuity equation:

$$
\nabla \cdot(\rho \mathbf{V})=J
$$

- the momentum equation:

$$
\nabla \cdot(\rho \mathbf{V} \mathbf{V})+\nabla p=\nabla \cdot \boldsymbol{\tau}+J\left(\mathbf{V}_{p}-\mathbf{V}\right)+n_{p} \mathbf{F}_{p}
$$

- the mass fraction equations for $i$-th species:

$$
\nabla \cdot\left(\rho \mathbf{V} Y_{i}\right)=-\nabla \cdot \mathbf{J}_{i}+R_{i}+S_{i}
$$

- the energy equation:

$$
\begin{aligned}
& \nabla \cdot[\mathbf{V}(\rho h+p)]=\nabla \cdot\left(\lambda \nabla T-\sum_{j=1}^{n} \mathbf{J}_{j} h_{j}+\boldsymbol{\tau} \cdot \mathbf{V}\right) \\
& +\alpha A_{p} n_{p}\left(T_{p}-T\right)-\sum_{j=1}^{n} h_{j}^{0} R_{j}-J \cdot\left(1-f_{h}\right) h_{c h},
\end{aligned}
$$

- the equation of state:

$$
\rho=p / R_{u} T \sum_{i=1}^{n}\left(Y_{i} / M_{i}\right)
$$

where

V - the velocity; $p$ - the pressure; $\rho$ - the gas density; $\mathbf{J}_{i}$ - the vector of the diffusion flux of the i-th gas species, $\mathbf{J}_{i}=-\rho D_{i, m} \nabla Y_{i} ; M_{i}-$ the molar mass of the 
i-th gas species; $R_{u}$ - the universal gas constant; $\tau-$ the tensor of viscous stresses, $\boldsymbol{\tau}=\mu\left(\nabla \mathbf{V}+\nabla \mathbf{V}^{T}\right) ; D_{i, m}$ - the laminar diffusion coefficient of the i-th gas species; $R_{i}$ - the mass rate of $\mathrm{i}$-th species production by homogeneous chemical reactions $R_{i}=M_{i} \sum_{r=1}^{N_{r}} R_{r, i} ; n-$ the number of reagents for the i-th reaction; $S_{i}$-the mass rateof $i$-th species production by heterogeneous chemical reactions, $S_{i}=\sum_{r=1}^{M_{r}} S_{r, i} ; R_{r, i}-$ the mass rate of i-th species production by $r$-th homogeneous chemical reaction $R_{r, i}=v_{r, i} A_{r, i} \exp \left(-\frac{E_{1, i}}{R_{y} T}\right) \prod_{j=1}^{n}\left[c_{r, j}\right]^{\eta_{r, j}} ; E_{1, i}-$ the activation energy of $\mathrm{i}$-th homogeneous reaction; $c_{r, i}$ - the molar concentration of i-th species, $c_{r, i}=\rho Y_{r, i} / M_{r, i} ; S_{r, i}$-the mass rate of $\mathrm{i}$-th species production by $r$-th heterogeneous chemical reaction; $N_{r}$ - the number of homogeneous reactions, in which i-th species of gas mixture takes place; $M_{r}$ - the number of heterogeneous reactions, in which i-th species of gas mixture takes place; $n_{p}$ - the number of particles per unit volume; $h-$ the enthalpy, $h=\sum_{j=1}^{n} Y_{j} h_{j}+\frac{\mathbf{V}^{2}}{2}$, $h_{j}=\int_{T_{0}}^{T} c_{p, j} d T, T_{0}=298.15 ; h_{j}^{0}-$ the enthalpy of formation for $\mathrm{j}$-th species; $\lambda$ - the coefficient of thermal conductivity; $Y_{i}$ - the mass fraction of i- species; $J-$ the mass rate due to the combustion of particles, $J=-n_{p} \frac{d m_{p}}{d t}$.

The system of equations describing the motion of reacting carbon particles includes

- the equation for the particle's coordinate:

$$
\frac{d \mathbf{x}_{p}}{d t}=\mathbf{V}_{p}
$$

- the equation for the particle's velocity:

$$
m_{p} \frac{d \mathbf{V}_{p}}{d t}=\mathbf{F}_{D}
$$

- the equation for the particle's temperature

$$
m_{p} c_{p} \frac{d T_{p}}{d t}=\alpha A_{p}\left(T-T_{p}\right)-f_{h} \frac{d m_{p}}{d t} h_{c h}
$$

- the equation for the particle's mass

$$
\frac{d m_{p}}{d t}=\frac{d m_{c h a r}}{d t}+\frac{d m_{v o l}}{d t}
$$

where

$m_{p}$ - the particle mass; $\mathbf{F}_{p}$ - the particle drag force $\mathbf{F}_{p}=3 \pi \mu d_{p} f_{D}\left(\mathbf{V}-\mathbf{V}_{p}\right) ; f_{D}$ - the particle drag force function [15]; $m_{c h a r}-$ the char mass of particle; $m_{v o l}-$ the volatile mass of particle; $c_{p}$ - the specific heat of the particle material; $A_{p}$ - the particle surface area, $A_{p}=\pi d_{p}^{2} ; \alpha-$ the heat transfer coefficient, $\alpha=\frac{\lambda}{d_{p}}\left(2.0+0.6 \operatorname{Re}_{p}^{1 / 2} \operatorname{Pr}^{1 / 3}\right) ; \quad \operatorname{Re}_{p}$ - the Reynolds number for particle, $R e_{p}=\frac{\rho d_{p}\left|\mathbf{V}_{p}-\mathbf{V}\right|}{\mu} ; \operatorname{Pr}-$ the Prandtl number, $\operatorname{Pr}=\frac{{ }^{c_{p} \mu}}{\lambda} ; h_{c h}-$ the heat released by the surface reaction; $f_{h}$-the fraction of the surface reaction heat absorbed by the particle; $f_{c o m b}-$ the combustible fraction of particle.

The model of coal particle combustion is assumed to be as follows. The particle does not change its mass if its temperature is less than the evaporation temperature of volatiles $T_{p}<T_{\text {vap }}$. After reaching the temperature $T_{\text {vap }}$ the mass of the particle decreases until it becomes less than $\left(1-f_{v, 0}\right) m_{p, 0}$ according to the law:

$$
\frac{d m_{v o l}}{d t}=-A_{0} f_{v, 0} m_{p, 0}, \frac{d m_{c h a r}}{d t}=0
$$

where $f_{v, 0}$ - the volatile fraction of particle at the initial time, $0.129 ; m_{p, 0}-$ the initial mass of particle; $A_{0}$ - the volatile rate constant, $50 \mathrm{~s}^{-1}$.

During the entrainment of volatile substances, the particle size changes and is determined as follows:

$$
d_{p}=d_{p, 0}\left(1+\left(C_{s w}-1\right)\left(m_{p, 0}-m_{p}\right) / f_{v, 0} m_{p, 0}\right)
$$

where $C_{s w}$-the swelling coefficient, $1.4 ; d_{p, 0}-$ the initial diameter of particle.

After evaporation of the volatiles, a heterogeneous oxidation reaction takes place between the carbon of the particle and oxygen to form carbon dioxide and water vapor:

$$
\begin{gathered}
\left(1-f_{v, 0}-f_{\text {comb }}\right) m_{p, 0}<m_{p}<\left(1-f_{v, 0}\right) m_{p, 0}: \\
\frac{d m_{c h a r}}{d t}=\sum_{i=1}^{3} S_{i}, \frac{d m_{v o l}}{d t}=0
\end{gathered}
$$

where

$S_{i}$-the mass rate of the particle due to a heterogeneous reaction with the $i$-th oxidizing species:

$$
S_{i}=A_{p} p_{i} \frac{R_{\text {dif }, i} R_{k i n, i}}{R_{\text {dif }, i}+R_{\text {kin }, i}}
$$


$p_{i}$ - the partial pressure of the i-th oxidizing species; $R_{\text {dif }, i}$ - the diffusion rate of the i-th oxidizing species to the particle surface,

$$
R_{\text {dif }, i}=\frac{C_{i}}{d_{p}}\left[\frac{\left(T_{p}+T\right)}{2}\right]^{0.75}
$$

$R_{\text {kin, } i}$ - the kinetic rate of the $\mathrm{i}$-th heterogeneous reaction,

$$
R_{k i n, i}=B_{i} \exp \left(-\frac{E_{2, i}}{R_{y} T_{p}}\right)
$$

$E_{2, i}$ - the activation energy of the i-th heterogeneous reaction.

The temperature of the inner walls is defined by the Laplace equation:

$$
\Delta T=0
$$

Boundary conditions. The velocity, the temperature of the gas, the mass concentrations of methane and oxygen are set at the entrance to the burner. The velocity and temperature of the particles are set equal, respectively, to the velocity and temperature of the gas. A pressure equal to atmospheric pressure is given at the outlet of the burner. The diffusion flux and the heat flux are set equal to zero on the heat-insulated walls. The non-slip condition for the gas is set on the walls. At the boundary of the gas mixture - internal walls, the equality of temperatures and the equality of heat fluxes are set.

\section{Numerical Description}

The system of equations for the gas phase is solved numerically by the Patankar method using an upwind scheme of second-order accuracy for the convective terms of the equations. The system of equations for particles is solved by an improved Euler method of second-order accuracy. The implementation of difference schemes is realized using the Ansys Fluent package [16].

The grid of 24000 quadrilateral cells is taken to cover the computational domain with 34 cells located across each burner channel.

The algorithm for obtaining a numerical solution includes three stages: 1) the solution of the system of equations for a gas phase not taking into account chemical reactions and particles; 2) specifying the temperature of the inner walls of $1000 \mathrm{~K}$ and solving the system of equations for the gas, taking into account chemical reactions in a gas phase without particles; 3) joint solution of the system of equations for gas phase and particles, taking into account chemical reactions for gas and particles.

\section{Results and Discussion}

Calculations were carried out with the following values of the parameters: the burner channel width $-6 \mathrm{~mm}$, the thickness of the inner walls of the burner $-0.2 \mathrm{~mm}$. The thermophysical parameters of the wall corresponded to the parameters of stainless steel. At the entrance to the burner, the following parameters were set: $Y_{\mathrm{O}_{2}}=0.2298$, $Y_{\mathrm{CH}_{4}}=0.01122, Y_{N_{2}}=0.75898$ that corresponds to $2 \%$ methane-air mixture, $T_{i n}=300 \mathrm{~K}$, the velocity $U_{i n}$ was variable. Particle size $d_{p}=4 \mu \mathrm{m}$. The rate constants of chemical reactions are given in Tables 1 and 2 .

The system of equations describing of the gas phase parameters was solved by the Patankar method using the up-wind scheme of second-order accuracy in space for the convective terms of the equations. The system of equations for particles was solved by an improved Euler method of second-order accuracy. The system of equations for particles was solved by an improved Euler method of second-order accuracy: 1) Solution is a system of equations for gas without taking into account chemical reactions and particles; 2) Specifying the internal wall temperature to $1000 \mathrm{~K}$ and solving the system of equations for the gas with allowance for chemical reactions without particles; 3) Solution of the system of equations for gas and particles.

Table 1. The rate constants of homogeneous reactions.

\begin{tabular}{|c|c|c|c|c|}
\hline $\begin{array}{c}\text { Number } \\
\text { of } \\
\text { reaction }\end{array}$ & $\begin{array}{c}A_{i} \\
\text { (variable } \\
\text { dimension) }\end{array}$ & $\begin{array}{c}E_{i} \\
(\mathrm{~J} / \mathrm{kmole})\end{array}$ & $\eta_{1}$ & $\eta_{2}$ \\
\hline 1 & $1.6596 \times 10^{15}$ & $1.72 \times 10^{8}$ & 1.46 & 0.5217 \\
\hline 2 & $7.9799 \times 10^{14}$ & $9.654 \times 10^{7}$ & 1 & 1 \\
\hline 3 & $2.2336 \times 10^{14}$ & $5.1774 \times 10^{8}$ & 1 & - \\
\hline 4 & $2.119 \times 10^{11}$ & $2.027 \times 10^{8}$ & 0.2 & 1.3 \\
\hline 5 & $1 \times 10^{15}$ & $1 \times 10^{8}$ & 1 & 1 \\
\hline
\end{tabular}

Table 2. Constants in the combustion model of a particle [17].

\begin{tabular}{|c|c|c|c|}
\hline $\begin{array}{c}\text { Number } \\
\text { of } \\
\text { reaction }\end{array}$ & $\begin{array}{c}B_{i} \\
\left(\mathrm{~kg} / \mathrm{m}^{2} \mathrm{~s} \cdot \mathrm{Pa}\right)\end{array}$ & $\begin{array}{c}E_{i} \\
(\mathrm{~J} / \mathrm{kmole})\end{array}$ & $\begin{array}{c}C_{i} \\
\left(\mathrm{~s} / \mathrm{K}^{0.75}\right)\end{array}$ \\
\hline 6 & 0.005 & $7.4 \times 10^{7}$ & $4.13 \times 10^{-12}$ \\
\hline 7 & 0.00635 & $1.62 \times 10^{8}$ & $1.69 \times 10^{-12}$ \\
\hline 8 & 0.00192 & $1.47 \times 10^{8}$ & $4.12 \times 10^{-12}$ \\
\hline
\end{tabular}

Figures 2, 3 show the positions of the heat release zone of the chemical reaction as a function of the feed rate of the coal-methane-air mixture in the burner and the concentration of coal particles. An increase in the feed rate of the mixture leads to a shift in the heat release zone to the center of the burner, Figure 2. If the value of the feed rate of the mixture exceeds the limit value at which the heat release zone is displaced from the center of the burner towards the outlet channel, its position becomes unstable. In this case, the heat removal from the reaction zone towards the cold mixture becomes large and the combustion stops (the burner stops operating in the recuperative mode). An increase in the concentration 
of coal particles leads to a shift of the heat release zone to the channel inlet, thereby widening the boundaries of the stable operation of the burner, Figure 3. Calculations show that increasing the particle concentration from $0.167 \mathrm{~g} / \mathrm{m}^{3}$ to $16.7 \mathrm{~g} / \mathrm{m}^{3}$ leads to an increase in the critical feed rate of the mixture from $0.7 \mathrm{~m} / \mathrm{s}$ to $0.8 \mathrm{~m} / \mathrm{s}$.

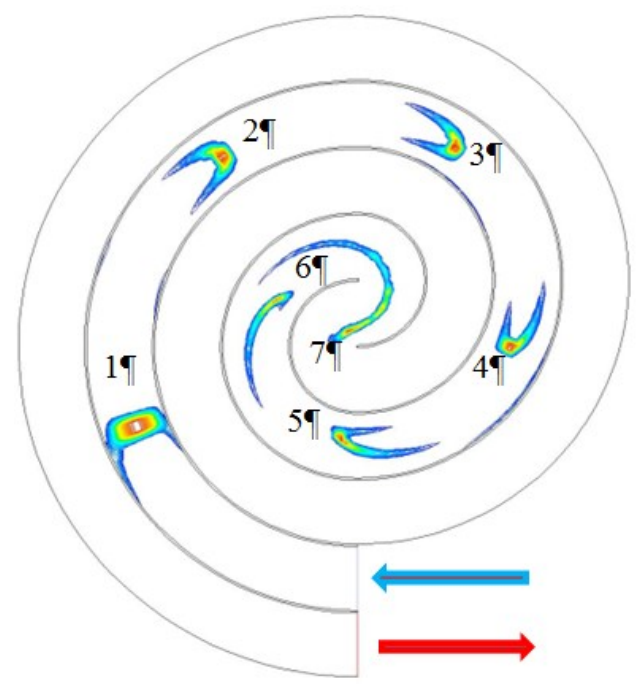

Fig. 2. Position of the heat release zone for low coal concentration

$1-U_{\text {in }}=0.1 \mathrm{~m} / \mathrm{s}, 2-0.2 \mathrm{~m} / \mathrm{s}, 3-0.3 \mathrm{~m} / \mathrm{s}, 4-0.4 \mathrm{~m} / \mathrm{s}$, $5-0.5 \mathrm{~m} / \mathrm{s}, 6-0.6 \mathrm{~m} / \mathrm{s}, 7-0.7 \mathrm{~m} / \mathrm{s}$; $\rho_{s}=1.67 \mathrm{~g} / \mathrm{m}^{3}$.

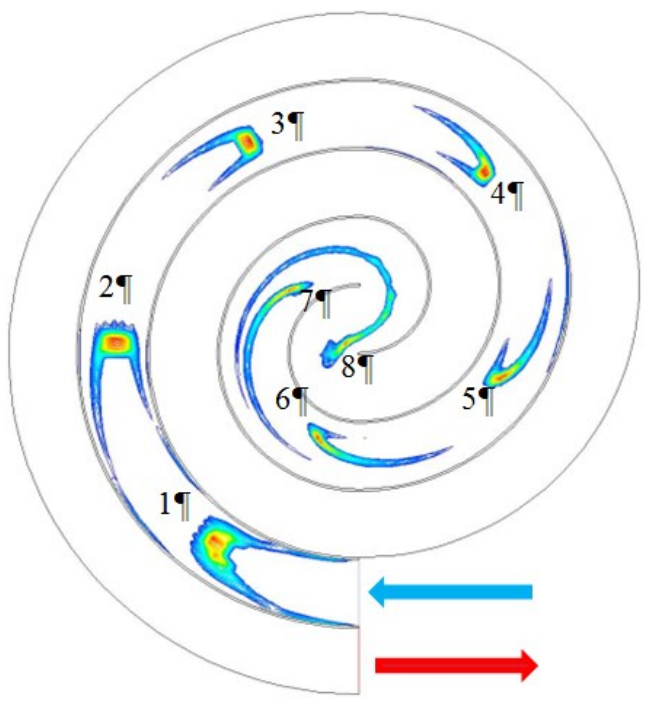

Fig. 3. Position of the heat release zone for high coal concentration

$1-U_{\text {in }}=0.1 \mathrm{~m} / \mathrm{s}, 2-0.2 \mathrm{~m} / \mathrm{s}, 3-0.3 \mathrm{~m} / \mathrm{s}, 4-0.4 \mathrm{~m} / \mathrm{s}$,

$5-0.5 \mathrm{~m} / \mathrm{s}, 6-0.6 \mathrm{~m} / \mathrm{s}, 7-0.7 \mathrm{~m} / \mathrm{s}, 8-0.8 \mathrm{~m} / \mathrm{s}$; $\rho_{s}=16.67 \mathrm{~g} / \mathrm{m}^{3}$.

An increase in the feed rate of the mixture leads to a decrease in the temperature of the combustion products at the outlet of the burner, Figure 4 . When the feed rate of a mixture is close to the maximum one, the maximum temperature is sharply reduced, Figure 5 that is caused by an increase in the removal of heat from the reaction zone to the cold mixture.

An increase in the concentration of coal particles leads to the fact that the heat release in the burner increases, leading to an increase in the maximum temperature and, as a consequence, to an increase in the temperature at the outlet of the burner, Figure 4.

An increase in the concentration of coal particles changes the behavior of the dependence of the maximum product temperature on the feed rate of the mixture, Figure 5. Calculation show that the maximum temperature increases with increasing the feed rate of the mixture at insignificant concentrations of coal particles $\left(0.16-1.67 \mathrm{~g} / \mathrm{m}^{3}\right)$, and then falls, reaching a maximum at a speed of $0.5 \mathrm{~m} / \mathrm{s}$. For a particle concentration of $16.7 \mathrm{~g} / \mathrm{m}^{3}$ and higher, the maximum temperature decreases monotonically with increasing feed rate of the mixture.

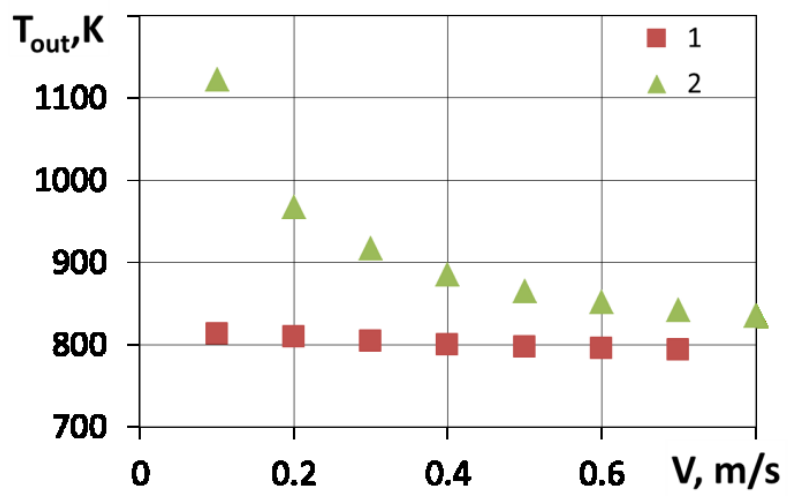

Fig. 4. Dependence of the combustion products temperature at the burner outlet on the feed rate of mixture $1-\rho_{s}=1.67 \mathrm{~g} / \mathrm{m}^{3}, 2-\rho_{s}=16.67 \mathrm{~g} / \mathrm{m}^{3}$.

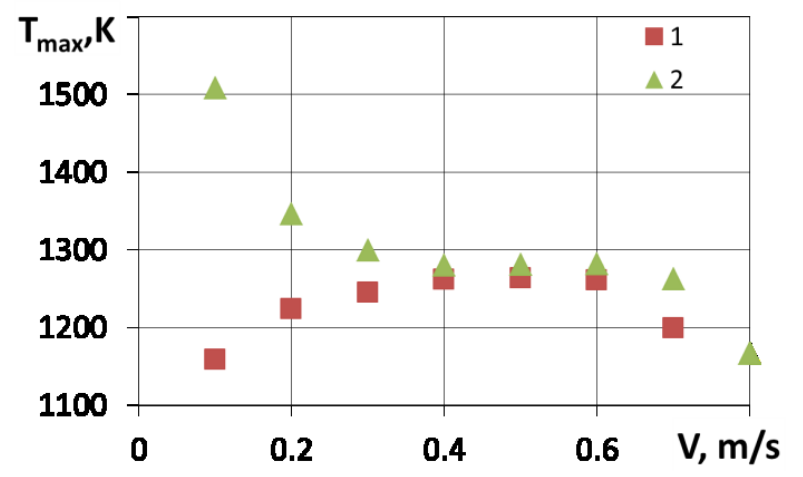

Fig. 5. Dependence of the maximum temperature of the combustion products on the feed rate of the mixture $1-\rho_{s}=1.67 \mathrm{~g} / \mathrm{m}^{3}, 2-\rho_{s}=16.67 \mathrm{~g} / \mathrm{m}^{3}$.

An increase in the feed rate of the mixture leads to an expansion of the region of penetration of the char residue in the burner at the same concentration of coal particles. An increase in the content of the coal particles in the mixture at the same feed rate leads to an increase in temperature, which in turn increases the combustion rate of the coal particles and thereby reduces the area of the unburned char residue, Figures 6, 7 . 

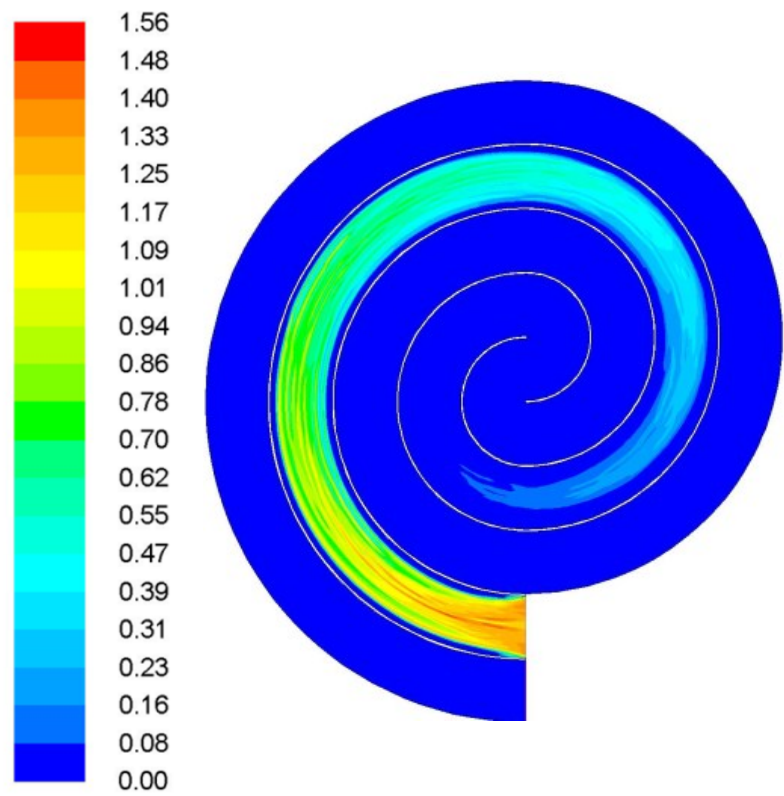

Fig. 6. Concentration of char residue $\rho_{s}=1.67 \mathrm{~g} / \mathrm{m}^{3}$.
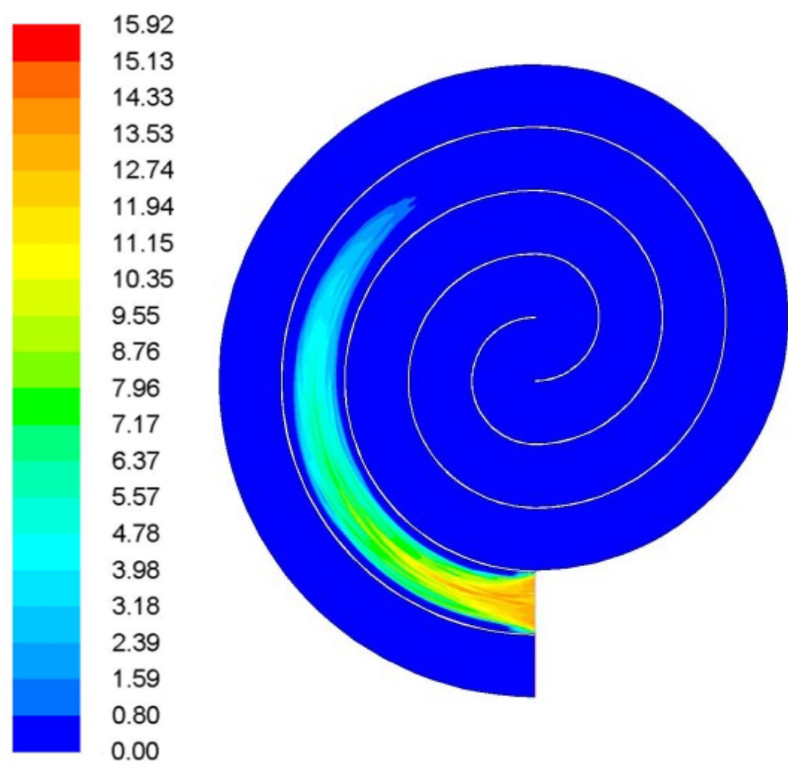

Fig. 7. Concentration of char residue $\rho_{s}=16.67 \mathrm{~g} / \mathrm{m}^{3}$.

\section{Conclusion}

The presence of coal particles in a lean methane-air mixture expands the range of stable operation of the "Swiss-roll" burner.

Increasing the content of coal particles reduces the combustion time of the particles due to increasing the combustion temperature.

The increase in the content of coal particles changes the shape of the reaction zone and shifts it to the inlet of burner.

An increase in the content of carbon particles leads to a change in the dependence of the maximum temperature on the feed rate of the mixture.

An increase in the content of carbon particles leads to a change in the dependence of the maximum temperature on the feed rate of the mixture.

This work was supported by the Russian Science Foundation grant No.17-79-20011.

\section{References}

1. E.G. Kovach, Technology of efficient energy utilization (Pergamon Press, Oxford, 1974)

2. F.J. Weinberg, Nature 233, 239 (1971)

3. J. Vican, B.F. Gajdeczko, F.L. Dryer, D.L. Milius, I.A. Aksay, R.A. Yetter, Proc. Combust. Inst. 29, 909 (2002)

4. A.C. Fernandez-Pello, Proc. Combust. Inst. 29, 883 (2002)

5. J. Chen, L. Yan, W. Song, D. Xu, Appl. Therm. Eng. 115, 702 (2017)

6. J. Chen, W. Song, D. Xu, Energy Convers. Manage. 134, 197 (2017)

7. A.Y. Krainov, K.M. Moiseeva, Combust. Explos. Shock Waves 52, 45 (2016)

8. A.Y. Krainov, K.M. Moiseeva, J. Eng. Phys. Therm. 89, 449 (2016)

9. V.N. Kurdyumov, M. Matalon, Proc. Combust. Inst. 33, 3275 (2011)

10. A. Krainov, L. Minkov, K. Moiseeva, MATEC Web Conf. 72, 01050 (2016)

11. S. B. Mane-Deshmukh, A. Krishnamoorthy, V. K. Bhojwani, Mater. Today: Proc. 5, 737 (2018)

12. Aiwu Fan, He Zhang, Jianlong Wan, Energy 123, 252 (2017)

13. K.M. Moiseeva, A.Yu. Krainov, D.M. Moiseev, Vestn. Tomsk. Gos. Univ.-Mat. Mekh. - Tomsk State Univ. J. Math. Mech. 48, 82 (2017)

14. A.Yu. Krainov, K.M. Moiseeva, Vestn. Tomsk. Gos. Univ.-Mat. Mekh. -Tomsk State Univ. J. Math. Mech. 3, 65 (2016)

15. S.A. Morsi, A.J. Alexander, Journal of Fluid Mechanics 55, 193 (1972)

16. ANSYS FLUENT 14.0 User's Guide. ANSYS, Inc., Southpointe, (2011)

17. W.P. Adamczyk, R.A. Bialecki, M. Ditaranto, P. Gladysz, N.E.L. Haugen, A. Katelbach-Wozniak, A. Klimanek, S. Sladek, A. Szlek, G. Wecel, Energy 140, 1305 (2017) 